\title{
Design and analysis of the tracker bridge for the Hobby-Eberly Telescope $^{*}$ wide field upgrade
}

\author{
Michael S. Worthington ${ }^{\mathrm{a} \dagger}$, Nicholas T. Mollison ${ }^{\mathrm{a}}$, Ian M. Soukup ${ }^{\mathrm{a}}$, Joseph J. Zierer ${ }^{\mathrm{a}}$, John M. Good ${ }^{\mathrm{b}}$, \\ Steven P. Nichols ${ }^{\mathrm{C}}$ \\ ${ }^{a}$ Center for Electromechanics, The University of Texas, 1 University Station R7000, \\ Austin, TX 78712 \\ ${ }^{\mathrm{b}}$ McDonald Observatory, The University of Texas, 1 University Station C1402, Austin, TX 78712 \\ ${ }^{c}$ Department of Mechanical Engineering, The University of Texas, 1 University Station C2200, \\ Austin, TX 78712
}

\begin{abstract}
A large structural weldment has been designed to serve as the new star tracker bridge for the Wide Field Upgrade to the Hobby-Eberly Telescope at McDonald Observatory in support of the Hobby-Eberly Telescope Dark Energy Experiment ${ }^{\ddagger}$. The modeling approach, analysis techniques and design details will be of interest to designers of large structures where stiffness is the primary design driver. The design includes detailed structural analysis using finite element models to maximize natural frequency response and limit deflections and light obscuration. Considerable fabrication challenges are overcome to allow integration of precision hardware required for positioning the corrector optics to a precision of less than 5 microns along the 4-meter travel range. Detailed descriptions of the bridge geometry, analysis results and challenging fabrication issues are discussed.

Keywords: Center for Electromechanics, Hobby-Eberly, HETDEX, tracker bridge, natural frequency, structural design

* The Hobby-Eberly Telescope is operated by McDonald Observatory on behalf of the University of Texas at Austin, the Pennsylvania State University, Stanford University, Ludwig-Maximillians-Universität München,and Georg-August-Universität, Göttingen

${ }^{\ddagger}$ http://hetdex.org/

${ }^{\dagger}$ m.worthington@cem.utexas.edu; phone 1-512-232-1670; http://www.utexas.edu/research/cem/
\end{abstract}

\section{INTRODUCTION}

The Hobby-Eberly Telescope (HET), Figure 1, is located on Mt. Fowlkes in the Davis Mountain Range in West Texas and has been conducting science operations since October, $1999^{1}$. The HET is a modified Arecibo-style telescope, with a 9.2-meter segmented primary mirror tilted at a fixed zenith angle of $35^{\circ 2}$. The star tracker mounts above the primary mirror at prime focus on the upper-most portion of the telescope, termed the upper hexagon or "upper hex". Corrector optics mounted to the tracker are positioned via two linear drive systems and a six degree of freedom (DOF) hexapod maintaining the instruments' optical axis normal to and on the focal sphere of the primary mirror.
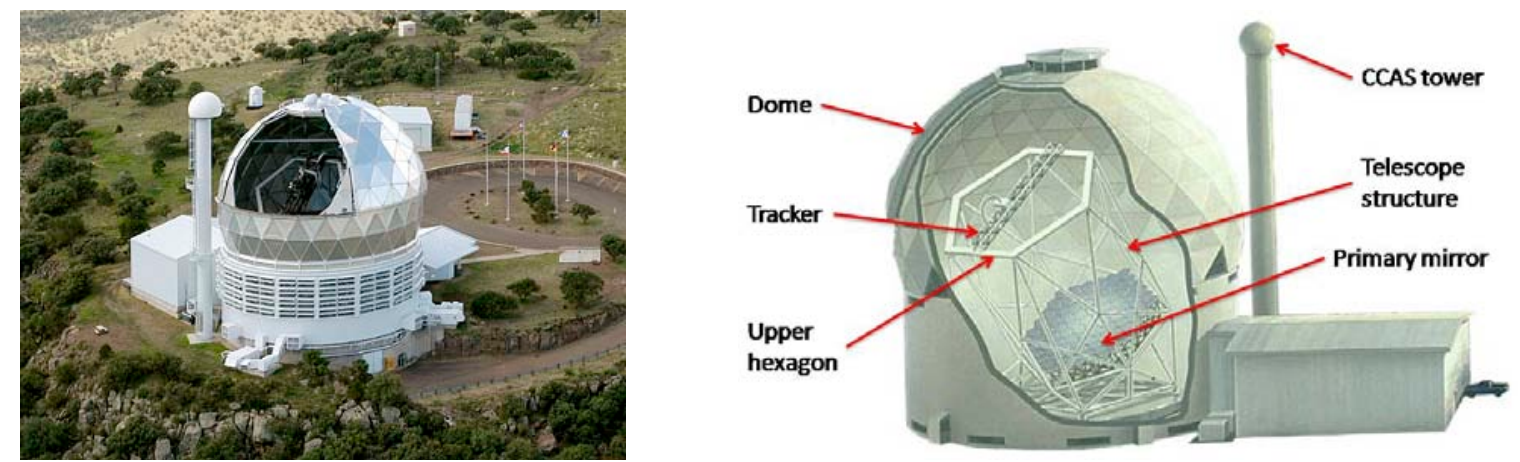

Figure 1. The photo on the left shows an aerial view of the HET with the shutter open. The computer rendering on the right reveals the major components of the telescope. 
The Hobby-Eberly Telescope is currently undergoing a major redesign effort in preparation for the Wide Field Upgrade (WFU) Dark Energy Experiment. The WFU involves replacing the current star tracker $^{3}$ along with its drive systems. The catalyst for replacing this hardware is to perform the Hobby-Eberly Telescope Dark Energy Experiment ${ }^{4,5}$ (HETDEX). Replacing the current Spherical Aberration Corrector (SAC) with the Wide Field Corrector ${ }^{6}$ (WFC) will increase the HET field of view from 4' to 22'. In addition, the current science instruments ${ }^{7}$ will be supplemented with the Visible Integral-field Replicable Unit Spectrograph ${ }^{8-10}$ (VIRUS). These changes will allow the telescope to conduct the largest survey of distant galaxies ever attempted.

The primary impact to the tracker caused by the replacement of the corrector optics for the WFU is the approximately seven fold increase in mass of the science instruments and supporting hardware. Due to the mass and geometry changes, the entire tracker, drive systems ${ }^{11,12}$, and control architecture ${ }^{13}$ are being replaced, including the bridge which serves as the backbone of the tracker. Experience with the HET tracker created new requirements for the WFU system which influences the bridge design, such as required frequency response, obscuration limits, and hexapod location.

This paper presents the methods used to design a large, welded structure whose primary requirement is a specified minimum resonant frequency. To understand the design choices made, such as the overall geometry of the bridge which greatly impacts the frequency response, details of the many functions that the bridge serves are presented. The modeling approach utilizes a number of interesting features to mimic the real world structure. Other designs can utilize these modeling techniques and, as a result, may be of particular interest to the reader. This also applies to the method of constraining degrees of freedom in the finite element analysis (FEA) software.

\section{TRACKER BRIDGE}

Throughout this paper, mention of the HET bridge refers to the existing bridge currently in operation whereas the WFU bridge is in direct reference to the new bridge. The term "payload" references all components mounted on top of the bridge that have the ability to translate the length of the bridge's main beams. The science payload is a subset of the overall payload and consists of the corrector optics necessary to capture and focus the light which reflects off of the primary mirror. Following is a description of the bridge functions and key telescope components mounted to the bridge.

\subsection{Bridge functions}

The tracker translates along two beams of the upper hex along the $\mathrm{X}$-axis via a drive system consisting of a pair of planetary roller screws and linear bearings ${ }^{11}$ shown in Figure 2. A second pair of linear bearings mounts on top of the two main bridge beams allowing the payload to translate in the $\mathrm{Y}$-axis via a third roller screw ${ }^{12}$. The $\mathrm{Z}$-axis is normal to the upper hex and main bridge beams, which is 35 degrees from the gravity vector. The telecentric optical axis, or Waxis, is the corrector's optical axis which tips and tilts to remain on a spherical focal surface matching the surface of the primary mirror. When the tracker is centered over the primary mirror, the $\mathrm{Z}$ and $\mathrm{W}$ axes are coincident. Manipulating the corrector optics also requires three rotational DOF. These DOF are theta (rotation about the X-axis, or tip), phi (rotation about the Y-axis, or tilt), and rho (rotation about the W-axis).
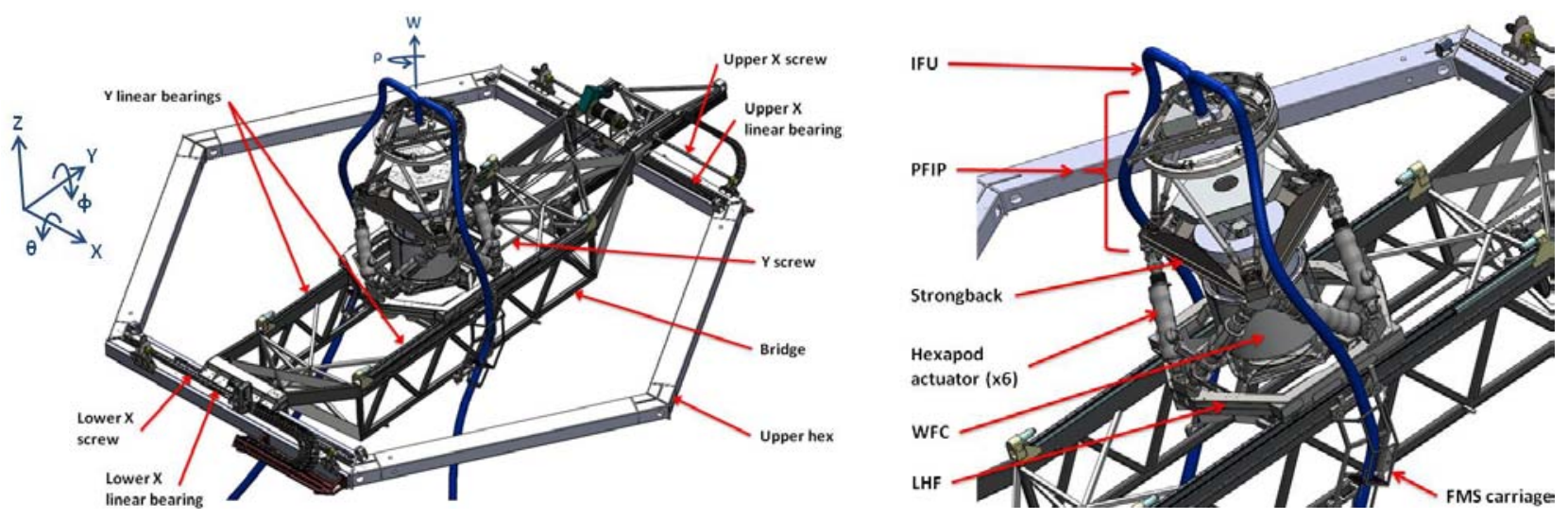

Figure 2. CAD model of the WFU tracker showing the key elements of the $\mathrm{X}$ and $\mathrm{Y}$ drive systems and the reference coordinate system (left image) and a close-up of the key components constituting the bridge payload. 
The bridge supports the payload mass which consists of several key components, also illustrated in Figure 2 with corresponding masses shown in Table 1 . Not shown in this figure are the many additional instruments, sensors, shrouds, cables and hoses that exist as part of the final payload. While the analyses account for the masses and locations of these components, the illustration shows a simplified view to provide clarity. The WFC is the first optical instrument which acts to focus light reflected from the primary mirror. The entrance pupil of the WFC must be positioned exactly at the primary mirror's prime focus and be aligned to the mirror's principal axis. As the telescope tracks a star, the tracker slowly moves in a combination of its six DOF. In addition to the $\mathrm{X}$ and $\mathrm{Y}$ drive systems, a hexapod provides six degrees of freedom (limited translations in X, Y and W and the three rotational DOF) to the WFC and the subsequent corrector optics mounted above, collectively named the Prime Focus Instrument Package (PFIP).

Table 1: Comparison of HET and WFU component masses: science payload consists of IFU loading through SAC/WFC and the bridge payload consists of every component except the bridge mass.

\begin{tabular}{|l|c|c|c|c|c|c|c|c|c|c|}
\hline Component & $\begin{array}{c}\text { IFU } \\
\text { loading }\end{array}$ & $\begin{array}{c}\text { Rotary } \\
\text { stage }\end{array}$ & PFIP & $\begin{array}{c}\text { SAC / } \\
\text { WFC }\end{array}$ & $\begin{array}{c}\text { Hexapod } \\
\text { system }\end{array}$ & $\begin{array}{c}\text { Carriage } \\
\text { / LHF }\end{array}$ & Y-drive & Misc. & Bridge & $\begin{array}{c}\text { Total } \\
\text { tracker }\end{array}$ \\
\hline HET [kg] & N/A & 70 & 350 & 90 & 139 & 573 & 80 & 182 & 2154 & $\mathbf{3 6 3 8}$ \\
\hline WFU [kg] & 260 & 987 & 1126 & 953 & 2929 & 2300 & 1510 & 631 & 8187 & $\mathbf{1 8 8 8 3}$ \\
\hline
\end{tabular}

The hexapod actuators ${ }^{14}$ mount to the Lower Hexapod Frame (LHF) and the strongback, which also supports the WFC. The PFIP mounts above the strongback, and is comprised of optical components and instruments in support of HETDEX and other programs. The light finally enters a group of 96 Integral Field Units ${ }^{15}$ (IFU) which house fiber optic cables. Light passes through the cables and is then analyzed in one of 96 VIRUS pairs. IFUs are a major addition to the original HET design. The impact of HETDEX with respect to the bridge is threefold: 1) the bridge must support additional hardware to route the IFUs towards their respective spectrograph cabinets, called the Fiber Management System ${ }^{16}$ (FMS); 2) the sum total of the fiber optic cables and their sheathing causes a significant loading not present on the original tracker; 3 ) the IFU bundles cause concern of additional loading during high wind conditions which may excite a natural frequency of the telescope.

The components of the FMS that interfaces with the bridge are the pair of carriages that mount to the sides of the bridge. The carriages consist of stress relieving hardware to prevent additional strain on the IFUs' connection point at the top of the PFIP and acts as a guide to minimize swinging loads as the payload translates. Each carriage rides on a pair of linear bearings allowing the carriage to translate with the payload. One bearing rail mounts to the outside of the bridge's main beams and the other mounts underneath the lower beams of the bridge on both sides.

\subsection{Design Constraints}

The absolute design driver for the WFU bridge is the $10 \mathrm{~Hz}$ minimum first mode natural frequency requirement. FEA reveals the entire telescope's lowest resonant frequency with the WFU tracker mounted on top of the upper hex is 5.35 Hz. Safe telescope operation requires the bridge's first mode be significantly greater than the overall telescope's first mode and subsequent experience with the HET proves the tracker is sufficiently stable at this design value. Given the substantial increase in payload, this requirement became increasingly difficult to meet.

The other key performance requirement for the bridge that the payload mass directly impacts is the deflection of the bridge beams as the payload traverses the length of the bridge. Since the WFC entrance pupil must always remain on the focal surface, the hexapod must compensate for deflections in the bridge. System requirements dictate a maximum bridge deflection of $2 \mathrm{~mm}$. Calculating this value requires subtracting the bridge's self-weight deflection with no payload from the worst case Z-axis deflection in the bridge beams under the full payload.

Both of the above requirements are to be met while keeping bridge mass and obscuration to a minimum. Obscuration is a measure of the amount of light being blocked by the tracker and can be visualized as the size of shadow being cast onto the primary mirror. Obscuration is typically expressed as a percentage of mirror area being obscured. Both of these parameters influence the choices of beam form factors and placement throughout the design. A number of constraints on the bridge design are dictated by the existing telescope structure. The following list includes the more pertinent constraints: 
- Bridge mounts at $35^{\circ}$ spanning the upper hex

- Distance between the upper and lower beams of the upper hex $(10,647 \mathrm{~mm})$ establishes bridge length

- Length of upper hex sides (6143 $\mathrm{mm}$ ) and required travel range $(4040 \mathrm{~mm})$ limits bridge width

- Required travel in Y-axis dictates length of bridge truss sections

- Primary mirror's radius of curvature establishes key Z-axis dimension for locating WFC entrance pupil (13.02 meters), establishing main bridge beam height in conjunction with design of the hexapod

Other constraints were created based on operational experience with the HET bridge. In the original HET design the hexapod is nested within the main trusses. One of the more significant constraints affecting the WFU bridge design is the requirement to locate the hexapod actuators above the bridge for more direct loading of the hexapod on the bridge beams and bearings, improved hexapod range of motion and greater maintenance accessibility. The impacts of this requirement include:

- Main bridge trusses must drop below the surface of the upper hex in order to maintain WFC location with respect to the primary mirror; results in a bridge more closely resembling the bridge at HET's sister telescope, the Southern African Large Telescope ${ }^{17}$ (SALT).

- The center of gravity (CG) of the new, taller and heavier payload rises substantially above the bridge's main beams due to the new design. This requires larger Y-axis linear bearings to counteract the tilting moment as the hexapod swings the science payload through its angular range of travel on the tracking sphere.

- The upper and lower mounts of all six actuators lie on a circle. To provide the most optimal load transfer from the actuators to the LHF and ultimately to the bridge, four actuator mounts align directly above the main bridge beams (illustrated in the second image of Figure 2). This couples the width of the bridge directly to the diameter of the hexapod mounting circle.

\section{SOLID MODELING}

\subsection{Bridge design}

The solid modeling portion of the design utilizes SolidWorks, a 3D mechanical computer aided design (CAD) software package. The structural and frequency analyses are performed using SolidWorks built-in FEA platform, Simulation (formally called CosmosWorks). The SolidWorks/Simulation package provides a key advantage in that it utilizes a single model for both the design effort and for analysis which is particularly important for inherently iterative designs such as the bridge. As the Simulation analyses identify improvements, or as the design adapts to other components changing within the system, the solid model is updated and quickly reanalyzed.

The WFU bridge is constructed from six major subassemblies (two trusses, two bulkheads and two end cross-braces) from a collection of bars, plates and thin-wall hollow structural section (HSS) tubing, as illustrated in Figure 3. The "Xbracing" members (1x9 in. plates) and side runners tie the two cross-braces to the main trusses. Available travel on the upper hex limits the width of the cross-braces. Due to the difference $(848 \mathrm{~mm})$ between the cross-braces and hexapod actuator mounting circle, which defines the truss spacing, the side runners angle both upwards and in towards the bridge's centerline to join the cross-braces. The upper runners are $12 \mathrm{x} 4 \mathrm{x}^{3} / 8$ in. box tubes and the lower members are $1 \mathrm{x} 9$ in. plates. The members which dramatically increase stiffness are the $2 \frac{1}{2} \times 22^{1 / 2}$ in. "V-braces". These solid members provide the required stiffness and buckling properties while providing substantially reduced obscuration than standard HSS members that have the same mass.

A major difference between tracker designs is the mounting arrangement of the Y-axis roller screw. The HET Y-screw mounts directly above the primary (wider) beam of the bridge. This ideal placement allows the screw to hide in the shadow of the primary truss, preventing additional obscuration. Originally, the intent for the WFU bridge was to mimic the HET Y-screw location but two problems were encountered. First, mounting the screw over the primary beam requires a significantly stiffer beam to react the moment from the payload about the Z-axis. As a result, the beam becomes heavier and obscuration increases as the beam's form factor grows to achieve the greater stiffness. The second issue is the mounting orientation of the hexapod creates an interference with the screw at this location. Mounting the screw either inside or outside of the bridge, like the SALT design, was considered but the modal performance issue was most important and the mechanical interference issue forced the screw into the light path regardless of these two options. 
This led to the center pull Y-drive, as shown in Figure 3 and further described in Reference 12, which not only raises the first mode frequency but also leads to a more desirable mode shape.

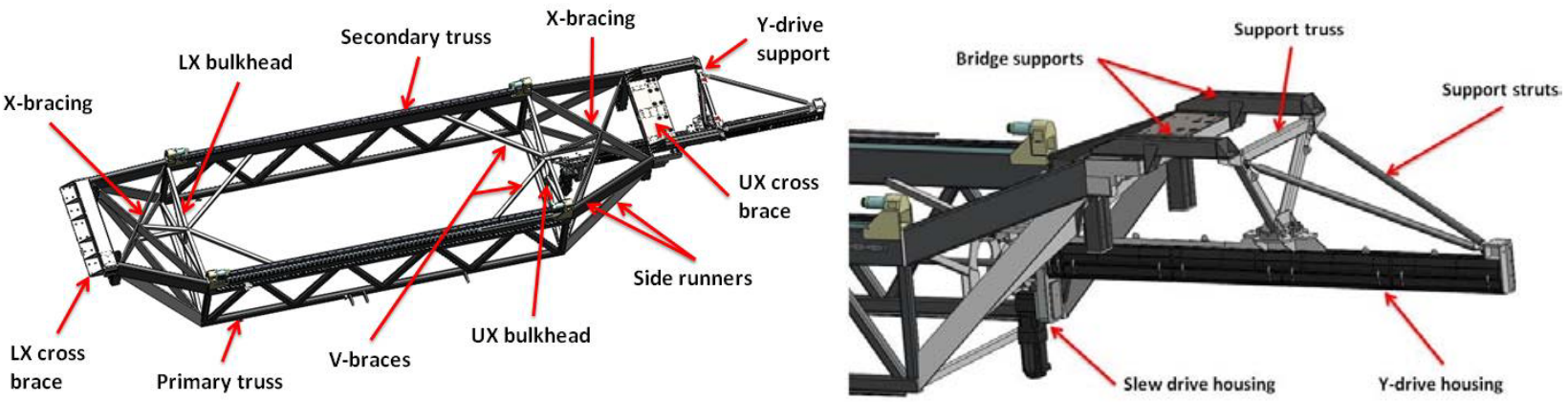

Figure 3: Major bridge subassembly and component identification (left) and Y-drive support structure details (right)

\subsection{Key features and geometry}

The bridge's main beams are each constructed with a pair of rectangular box tubes, Figure 4 . The primary beam consists of two $12 \mathrm{x}^{4} \mathrm{x}^{5} / 16$ in. tubes welded together lengthwise while the secondary beam is fabricated from two $12 \mathrm{x}^{2} \mathrm{x}^{1 / 4} \mathrm{in}$. tubes. Due to the load supported by the linear bearing rails, the main bridge beams are doubled so as to provide a stiffening member beneath the rails. A single $12 x 8$ in. beam on the primary side would require substantially thicker walls to prevent deflection across the width of the beam. Similar to the HET bridge, it was felt that having one beam structurally dominate the other would still provide a benefit even with the center pull Y-drive. The Y-axis linear bearings on the secondary rail have an additional DOF in the X-direction decoupling the secondary rail from reaction forces. As a result, the primary beam bearing blocks react these forces to the bridge through the primary rail. Bar stock welded along the top of both main beams provide material for machining the linear bearing way details. The box tubing walls are too thin to directly bolt the rails thereby providing an additional requirement for this added material. Bar stock on the primary beam also has mounting features to support the linear encoder used to track the payload's position along the Yaxis. For similar reasons, additional stock material is welded and later machined to the outer side of the main beams to mount the smaller FMS linear bearings.
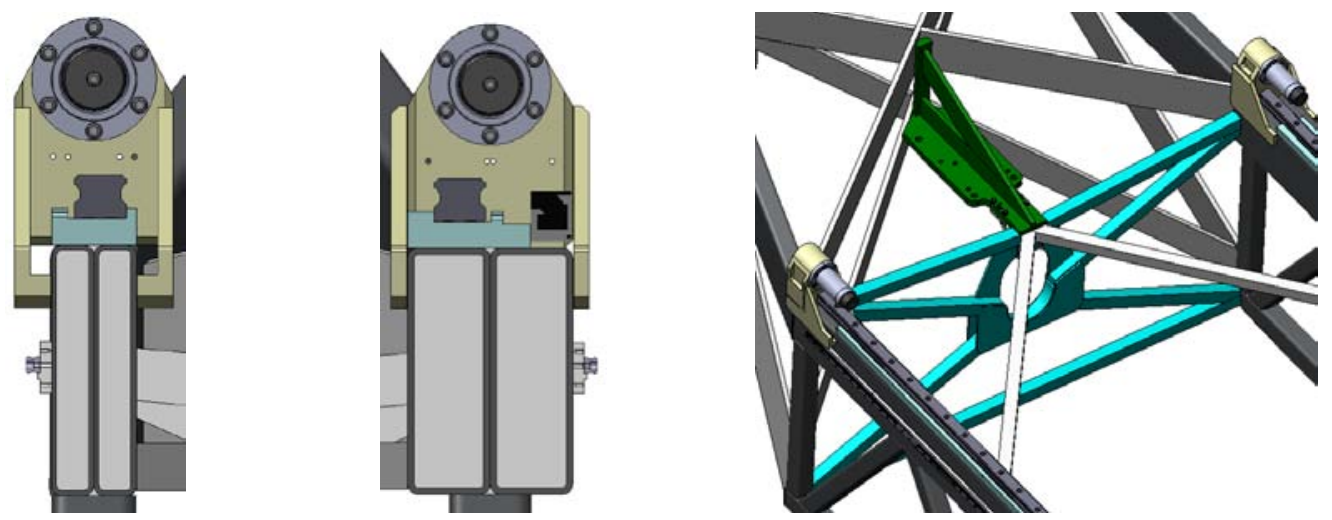

Figure 4: Section views of the secondary beam, left, and the primary beam, middle. Evident are the main linear bearing rails and ways (light blue), the FMS linear bearing rails and ways welded to the sides of the beams and the hard stop mounts in the background. The right image shows the upper bulkhead (blue) and mounting bracket (green) for the Y-drive, slew drive assembly and roller screw nut.

The lower beams of both main truss sections are constructed of $5 x 4 x^{3} / 8$ in. box tubes. Additional bar stock is welded and machined to the bottom of these beams to mount the other FMS linear bearing rails. The angled truss beams and the vertical beams at the ends of the trusses are $4 \times 4 x^{3} / 8$ in. tubes. Throughout the design iterations, the distance between the upper and lower truss beams was a variable. The primary penalty for adding height to these main trusses is extra mass. 
The bulkheads are primarily constructed of $2 \frac{1}{2}$ in. square bar with horizontal members spanning the top and bottom and additional members forming an "X" across the middle. Additional bars tie the bulkhead to the X-bracing. Upon adapting the center pull Y-drive configuration, the upper bulkhead, Figure 4, had to be modified to allow the roller screw to pass through. The bars tying the bulkhead to the X-braces were significantly modified in order to mount the slew drive housing which also contains the roller screw nut. This arrangement fixes the nut onto the bridge and allows the roller screw to translate with the payload.

As previously shown in Figure 3, the center pull Y-drive extends from the top of the Upper-X (UX) cross brace. The main supports are a pair of $6 \times 6$ in. HSS tubes, each constructed of two $6 \times 3 \times 1 / 4$ tubes welded together. These weldments bolt onto the bridge creating a modular assembly, allowing for simpler initial installation of the bridge onto the upper hex. The other ends of these bridge supports are joined via a support truss, a triangular group of $4 \times 3 \times 1 / 4$ in. tubes tying the braces to the main Y-drive support. In addition, two support struts, NPS 2 in. SCH 80 pipes, extend from the main braces out to the end of the Y-drive housing and attach via spherical rod ends. The Y-drive housing itself is a weldment made from a $2 x^{3} x^{1 / 4}$ in. tube with $13 / 4 x^{1 / 2}$ in. bar stock welded along the bottom side and a $2 x^{1 / 2}$ in. bar standing vertical welded to the upper side. The lower piece of bar stock serves as the way for a linear bearing. The roller screw bearing housing is bolted to the linear bearing block allowing the screw to translate with the payload. The sides of the housing have hinged access panels down the entire length for maintenance accessibility.

The end cross-braces of the bridge, Figure 5, have seen the most iterations throughout the bridge design. Formerly constructed entirely of rectangular box tubing, these subassemblies are now fabricated from an assortment of plates welded together. The Lower-X (LX) end has a "shelf" as part of the design to eliminate a significant interference with the X-drive slew and nut housing. This end also has provisions for mounting a bracket that ties to the LX cable handler which feeds numerous cables and hoses onto the bridge and in many instances onto the moving payload via the $\mathrm{Y}$-axis cable handler.

The UX cross-brace serves multiple functions. In addition to serving as the base for the Y-drive supports, the Constant Force Drive $^{12}$ (CFD) also mounts to the top plate of the cross brace. The upper most plate has multiple tapped hole patterns to fasten this hardware to the cross-brace. Additional hole patterns are provided on the front of the cross-brace to tie the Y-drive supports and on the back plate to attach the pulley for the CFD. Pockets are cut out of this plate and replaced with thinner steel plates to reduce weight while maintaining stiffness. Additional pockets are cut in the front section of the plate to pass bolts which tie the bridge to the X-drive linear bearings.
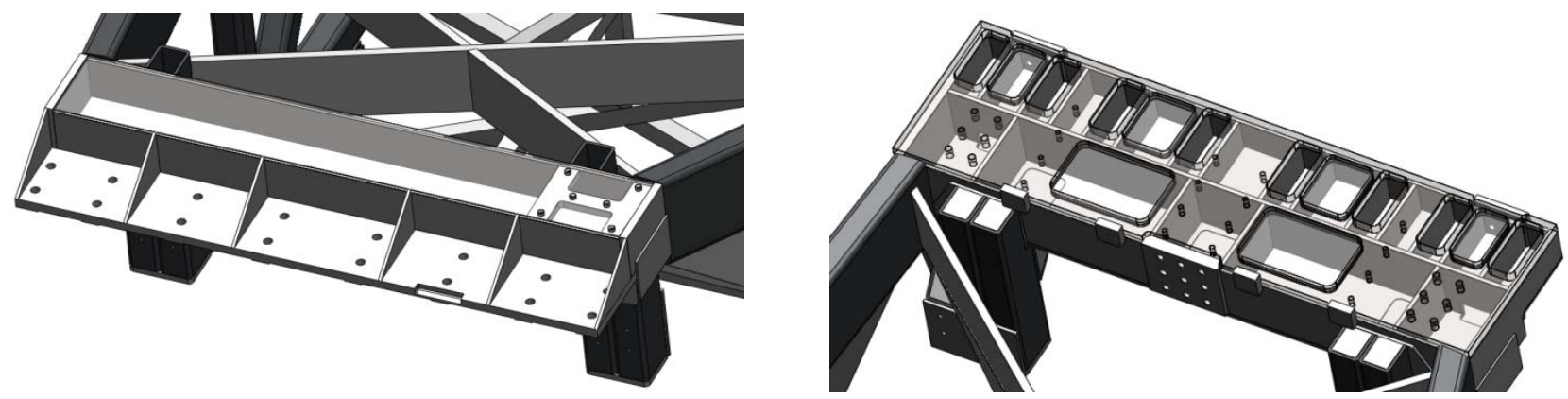

Figure 5: LX cross brace, left, with top plate shown semi-transparent to reveal the 'H' shaped LX cable handler bracket attachment point and the UX cross brace, right, with the top plates shown semi-transparent to reveal internal bracing.

\section{FINITE ELEMENT ANALYSIS}

Given that many of the bridge beams are HSS thin-walled tubes, attempting to perform a solid mesh with sufficient mesh density across the wall thicknesses over such a large structure would require considerable computing power and long run times. A beam mesh, however, converts the beam elements into an array of "stick figures" with the appropriate crosssectional properties. The result is a very quick running analysis ideal for this type of design. Figure 6 shows the meshed bridge ready for analysis.

The green arrows in the figure represent the constraints applied to the model. The four corners of the bridge are constrained in the Z-axis. At the center of the UX cross-brace, a constraint is applied in the X-direction preventing the 
bridge from translating down the length of the linear bearing. At this same location on the LX end, the bridge is constrained in both the $\mathrm{X}$ and $\mathrm{Y}$ directions. Reference 11 provides a detailed explanation of the bearing arrangements providing these DOF.

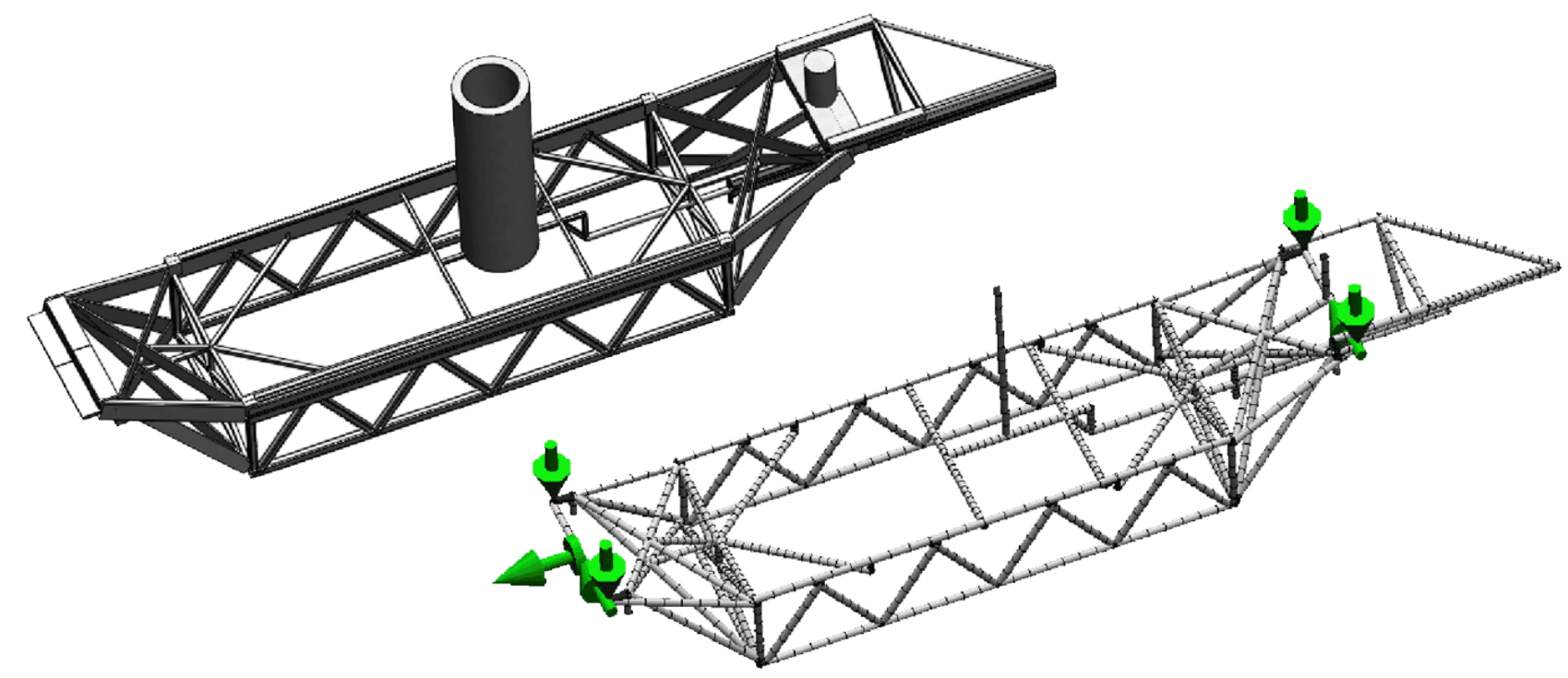

Figure 6: SolidWorks model and resulting Simulation mesh of the bridge with applied constraints shown (green arrows)

The model requires several manipulations to more accurately reflect the real bridge prior to analysis. The bridge will be in its final assembly state when it is mounted atop the upper hex and potentially excited by wind gusts. This means that in addition to the bridge itself, all subassemblies will be installed on the bridge. The main subassemblies are the payload, Y-drive and FMS carriages. These subassemblies are treated in various ways within the model:

- The $9044 \mathrm{~kg}$ payload is modeled as a hollow cylinder. The length of the cylinder is twice the height of the real payload's calculated CG to position the modeled payload's CG correctly. To represent the worst case loading condition, the payload's CG is positioned directly over the mid-span of the trusses, requiring the base of the payload to move upwards $900 \mathrm{~mm}$ along the $\mathrm{Y}$-axis due to the $35^{\circ}$ mounting angle.

- Locating the payload in the middle of the bridge is a mock LHF modeled as an " $\mathrm{H}$ " frame using $2 \mathrm{x}^{2} \mathrm{x}^{3} / 64 \mathrm{in}$. tubes. The Young's modulus of these tubes is artificially increased to represent the actual, stiff LHF. Beam releases are applied to the two members spanning between the trusses. On the primary side of the bridge, the beams are free to translate in the $\mathrm{Y}$-axis representing the $\mathrm{Y}$-axis linear bearings. On the secondary side, the LHF is free in both the $\mathrm{X}$ and $\mathrm{Y}$ axes.

- The Y-drive housing requires some modeling simplifications due to its complexity. The entire Y-drive subassembly, including the $60 \mathrm{~mm}$ diameter roller screw, has a mass of $913 \mathrm{~kg}$ and is represented by $926 \mathrm{~kg}$ of hardware in the analysis model due to the simplifications. More important than the mass is the location of the subassembly's CG and stiffness characteristics. The modeled Y-drive's CG is located within 2 percent of the real model in all three axes but matching the stiffness is much harder to control due to the limitations of beam modeling within SolidWorks.

- The four anti-skew blocks ${ }^{11}$ (165 kg each), four Y-axis hard stops ${ }^{11}$ (50 kg each), two FMS carriages (305 kg each), Y-drive slew housing ${ }^{12}(178 \mathrm{~kg})$ and CFD $(584 \mathrm{~kg})$ are all modeled as simple beams in their respective locations. The density and CG locations of each component are manipulated within the beam's properties to match the actual hardware.

Table 2 lists the key results obtained from the multiple FEA performed. While the $10 \mathrm{~Hz}$ first mode natural frequency could not be obtained due to the large mass supported, the final design is approved by McDonald Observatory. The following sections elaborate on the various analyses performed. 
Table 2: Summary of FEA results

\begin{tabular}{|l|c|c|c|}
\hline \multicolumn{2}{|l|}{ Parameter } & Units & Value \\
\hline \multirow{3}{*}{$\begin{array}{l}\text { Natural } \\
\text { Frequency }\end{array}$} & $\mathbf{1}^{\text {st }}$ & $\mathrm{Hz}$ & 9.40 \\
\cline { 2 - 4 } & $\mathbf{2}^{\text {nd }}$ & $\mathrm{Hz}$ & 10.72 \\
\cline { 2 - 4 } & $\mathbf{3}^{\text {rd }}$ & $\mathrm{Hz}$ & 13.70 \\
\hline Delta Deflection & $\mathrm{mm}$ & 1.506 \\
\hline Max Von Mises Stress & $\mathrm{MPa}$ & 48 \\
\hline \multicolumn{2}{|l|}{ Buckling Load Factor } & --- & -7.61 \\
\hline
\end{tabular}

\subsection{Natural frequency analysis}

The frequency response was the key design driver, making the frequency model the primary design tool. Simulation outputs mode shapes based on the analysis which can be viewed as animations. The first five mode shapes are studied, with the greatest attention paid to the first three. The first mode by definition has the lowest resonant frequency and is considered the most critical. Mode shapes indicate which areas of the bridge require stiffening to either change the mode shape or to raise the natural frequency within that shape. While the primary goal of the design process is to increase the first mode natural frequency, the design has to be optimized for multiple parameters. Overall mass and obscuration remain key considerations to monitor throughout the process. The linear bearings have load limitations to meet required life expectancy and while additional stiffness can be achieved using beams with greater cross-sectional properties, the obscuration produced would be counter-productive to the telescope's operation.

SolidWorks allows the user to scale results to more clearly depict mode shapes. The mode shapes and magnitudes for the first three modes are shown below with the deformation scales noted. Since the animations reveal so much more information than screen captures, the images below show the bridge at two separate intervals of the animation overlaid on top of one another. The images showing the bridge at the extremes are semi-transparent for clarity.

The first mode, Figure 7, measures $9.40 \mathrm{~Hz}$. Attempting to describe the mode shape, it is a combination of what is referred to within the team as the "snake" and "heave" modes. The snake mode creates an 'S' shape down the length of the bridge, as can be seen from the overhead view. The heave description references a vertical (Z-axis) flexing motion of the main bridge trusses that can be seen in the side view. In this particular mode, the primary bridge beam experiences greater displacement in both the positive and negative Z-directions. Raising this frequency would require stiffening the main bridge rails in both the $\mathrm{X}$-axis to reduce the snake mode and the $\mathrm{Z}$-axis to reduce heaving. This can be accomplished by utilizing larger box tubes and/or increasing wall thicknesses. Both of these changes will increase mass and possibly have a negative effect on obscuration.
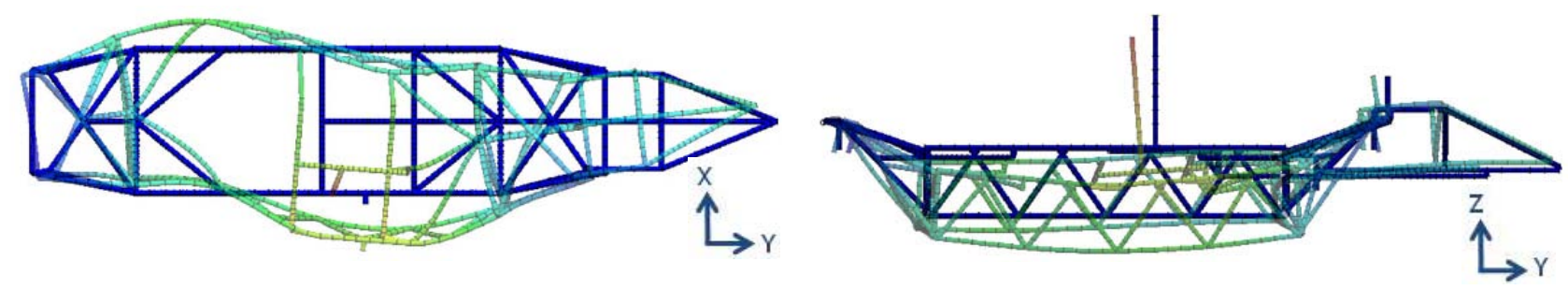

Figure 7: Overhead and side views of the first mode frequency response (deformation scale = 125)

The second mode, $10.72 \mathrm{~Hz}$ (Figure 8), behaves very similar to the first mode. The mode shape is again a combination of the snake and heave modes, except the secondary bridge beam experiences greater heave than the primary beam. Another difference is that in the second mode the Y-drive extending out from the bridge deflects in a similar manner as the UX end of the bridge, meaning the entire structure moves together. The Y-drive remained almost perfectly within the $\mathrm{X}-\mathrm{Y}$ plane in the first mode. The approach mentioned to stiffen the first mode should also contribute favorably in stiffening the second mode, albeit with similar consequences. 


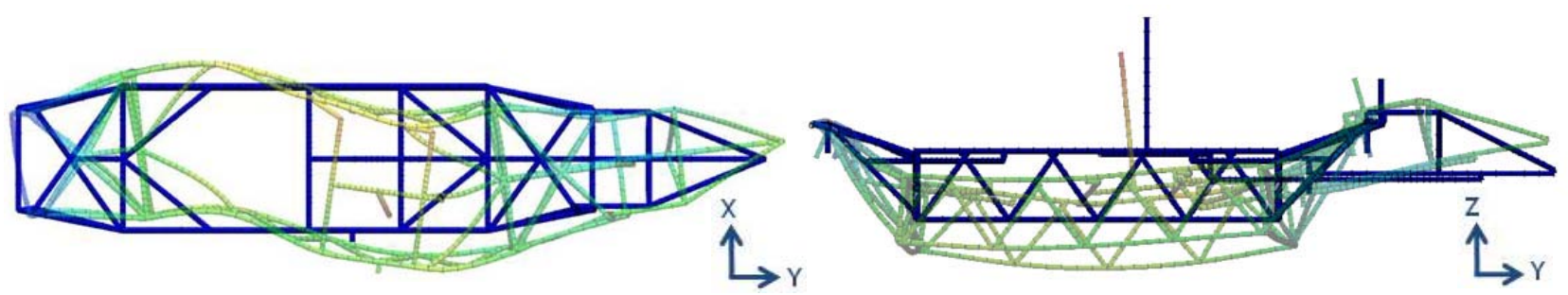

Figure 8: Overhead and side view s of the second mode frequency response (def. scale $=125$ )

The third mode, $13.70 \mathrm{~Hz}$, is also a heave mode but is limited almost entirely to the Y-drive and, to a lesser extent, the UX end of the bridge, Figure 9.

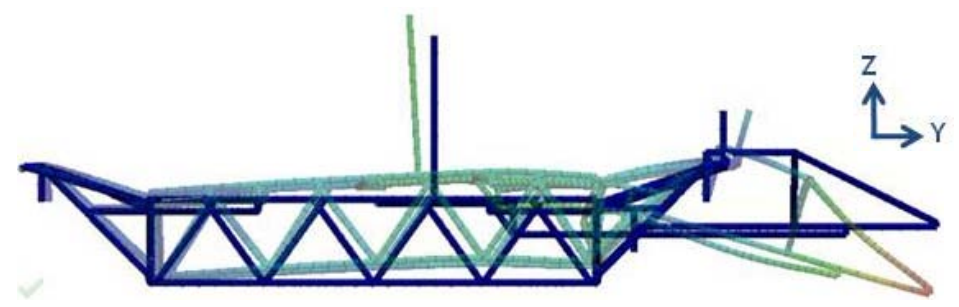

Figure 9: Side view of the third mode frequency response (def. scale $=55$ )

\subsection{Deflection analysis}

A separate, static analysis is performed to measure the deflection in the main bridge beams. The only difference between this model and the frequency model is the inclusion of gravity. The gravity vector is positioned 35 degrees from the Zaxis in the Y-Z plane. The WFU specification requires bridge deflection in the Z-axis under full payload be less than 2 $\mathrm{mm}$ with respect to deflection without the payload.

Without the payload, the maximum self-weight deflection measured along the Z-axis is $0.751 \mathrm{~mm}$. With the payload's CG positioned directly above the midpoint of the main bridge beams, the bridge measured $2.257 \mathrm{~mm}$ of deflection in the Z-axis, Figure 10. The resulting deflection caused by the $9044 \mathrm{~kg}$ payload is therefore the difference between these two measurements, $1.506 \mathrm{~mm}$. The location of greatest deflection is on the secondary beam near the joint with the lower cross member of the LHF. For comparison, the much lighter HET bridge reportedly has $0.991 \mathrm{~mm}$ of deflection ${ }^{5}$.

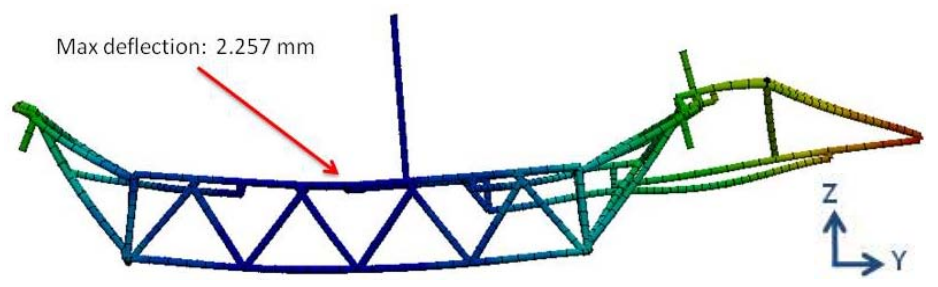

Figure 10: Bridge Z-axis deflection with the full payload resulting in maximum deflection of -2.257 mm (deformation scale $=300)$

\subsection{Stress analysis}

Stress and buckling analyses are also performed on the bridge to ensure all beam members are within acceptable safety limits. A minimum safety factor of 2.0 to yielding was established as a design rule for each member. The gravity vector is applied in both analyses providing the only source of loading to the bridge. The same constraints used in the deflection and frequency analyses are applied.

The worst case stresses are calculated by combining the axial and bending stresses about two axes at a critical point on the cross-section. The results show that beams with the highest stresses are in the LHF and the screw mount extending from the LHF. Given the drastic simplifications made to these LHF members, these stresses can be neglected and are 
studied in a separate analysis using the actual LHF geometry. Ignoring the LHF beams reveals a few areas that share the maximum stress. The center of the LX cross-brace measures approximately $48 \mathrm{MPa}$. The joints on the primary and secondary main beams of the bridge where the lower member of the LFH connects are two other areas with approximately $48 \mathrm{MPa}$ stress. In all three of these locations, the beams in question have a factor of safety with respect to yielding greater than 5 .

Despite the relatively low axial stresses within the model, a buckling analysis is performed as a safety check due to the use of many long, slender bars and plates. The results show that the weakest beam with respect to buckling is the $1 \mathrm{x} 9 \mathrm{in}$. lower runner from the primary truss to the LX cross-brace. The stress analysis reveals that this member experiences an axial stress of $11.2 \mathrm{MPa}$ resulting in an axial tensile load of approximately $65 \mathrm{kN}$. The calculated buckling load factor is -7.61. This means that the axial load would have to reverse (i.e. become a compressive load) and increase in magnitude by a factor of $7.61(\sim 495 \mathrm{kN})$ before this beam would buckle. These results reveal that buckling should not be a concern for this design.

\subsection{Validation}

After fabrication but prior to machining, an opportunity presented itself to perform a rap test on the bridge to experimentally determine the natural frequencies and mode shapes. The purpose of the test was to validate the analysis tools used in the design of the bridge. Since the bridge was in a different loading and constraint state than on the telescope, another FEA analysis was performed. This analysis assumed the bridge was supported in a free-free state, which was represented by hanging the real bridge by two lifting straps from overhead cranes.

The test utilized a single three-axis accelerometer mounted to the bridge and a roving instrumented impact hammer hitting the bridge along its length on both sides. The first mode results in a magnitude of $15.5 \mathrm{~Hz}$, compared to a predicted frequency of $14.6 \mathrm{~Hz}$. The second and third modes were also comparable: 17.9 and $20.1 \mathrm{~Hz}$ measured versus 18.0 and $22.6 \mathrm{~Hz}$ respectively. Given that the first mode does involve some vertical motion, it was expected that the measured response be slightly higher due to the contribution of the lifting straps not represented in the FEA model.

\section{FABRICATION}

A brief overview of the fabrication process is as follows. The stock material shapes are laser cut to size, many beams requiring angled, if not compound angled, cuts. Once all of the parts are prepared, welding on the subassemblies begins. The bulkheads tie the main trusses together and then numerous pieces tie the bridge cross-braces to the trusses including the X-braces and the side runners. Rectangular bar stock is welded to the bridge in areas where linear bearing rails are mounted. This stock material is later machined with the required mounting features for the rails. Additional items that weld onto the bridge are the two upper and two lower hard stops providing absolute limits for Y-travel, the four antiskew blocks which engage the upper hex to limit skewing and the mounting bracket for the Y-drive assembly. All welds must adhere to AWS D1.1:2008 standards and are accepted based upon the criteria for dye penetrant or magnetic particle inspection per the same standard.

Individual subassemblies are thermally stress relieved per SAE AMS 2759/11 during the fabrication process. Upon completion of all welding processes, the entire bridge is thermally stress relieved. This step is deemed of particular importance due to the amount of post-machining and tight tolerances required in the final component. Neglecting to stress relieve the bridge could result in parts deforming during the machining processes or could lead to the formation of cracks within the welds. All welds affected by machining operations will be re-inspected prior to delivery. Several key machining operations are required on the bridge:

- Bottom surfaces of the cross-braces which mount to the upper hex bearing stacks (these become datum surfaces referenced by other machining operations): flatness tolerance of $0.1 \mathrm{~mm}$ on the LX end and the UX end must be coplanar to the LX end within $0.1 \mathrm{~mm}$

- Top surface of the UX cross-brace for mounting the Y-drive support beams and CFD skid (flatness to $0.1 \mathrm{~mm}$ ), along with required bolt hole patterns

- Maintain parallelism between the main Y-axis linear bearing rails (refer to Figure 4 for a cross section view):

0 Bearing ways must be parallel to the cross-brace datum surfaces $( \pm 0.25 \mathrm{~mm})$ 
o Both rails have a machined datum shoulder for the full rail length $(6385 \mathrm{~mm})$ : primary rail shoulder must be perpendicular to the way surface $( \pm 0.25 \mathrm{~mm})$ and the secondary rail shoulder must be parallel to the primary shoulder $( \pm 0.10 \mathrm{~mm})$

o Second shoulder machined for each rail with tapped holes for pressing the rails against the datum shoulders with bolts

- Maintain parallelism between both pairs of FMS linear bearing rails: relaxed tolerances only require datum shoulder; shoulder and way parallelism tolerances $( \pm 0.75 \mathrm{~mm})$

- Y-axis linear encoder mounting surface (outward face of the primary rail bar stock): flatness requirement (0.05 $\mathrm{mm})$ and parallelism callout to the primary rail shoulder $( \pm 0.25 \mathrm{~mm})$

- Mounting surfaces for the Y-drive: for optimal roller screw performance and life, radial loads transmitted through the nut are not desired. Great care has therefore been given to ensure proper alignment between the roller screw axis and the $\mathrm{Y}$-axis bearing rails.

o Surfaces on Y-drive assembly mounting bracket and upper bulkhead are machined and holes are drilled to fasten a spherical bearing mount which is used to attach the Y-drive housing to the bridge

o Provisions for multiple adjustment features are also machined into the mounting bracket to aid in screw alignment

- Bolt hole patterns (drilled and tapped) for: attachment to X-axis linear bearings, CFD, Y-drive supports, linear encoder, all 6 bearing rails and two presser shoulders for the main bearing rails

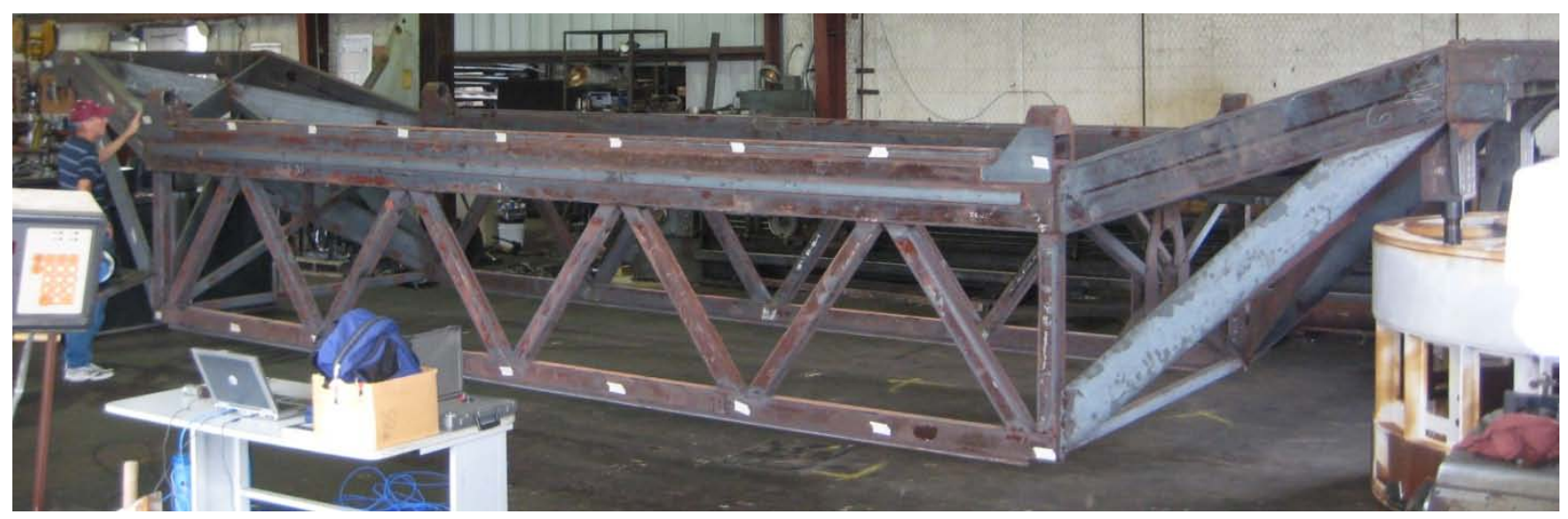

Figure 11: Photograph of the bridge after completion of fabrication and stress relieving, prior to machining

\section{SUMMARY}

The tracker bridge for the WFU was successfully designed and modeled utilizing 3D CAD software. Much of the exterior geometry of the bridge was dictated by the travel requirements and the hardware which the bridge must support. In addition to providing methods in which to support this hardware, the principle design effort created a very stiff but relatively lightweight structure. This is done while attempting to provide cost effective fabrication, limiting deflections and mass, and minimizing obscuration in the telescope optical path. Finite element analyses were performed on the bridge to study the natural frequency response, deflection, stresses and buckling. A vast collection of modeling techniques are used in the analyses which include using lumped masses to represent subassemblies, modifying the modulus of elasticity of specific beam members, and modeling DOF through the use of constraints and beam releases. Additional details of the modeling and analysis of the WFU bridge can be found in Reference 18.

The final first mode natural frequency of the bridge, $9.4 \mathrm{~Hz}$, came close to the original design goal of $10 \mathrm{~Hz}$. It was known that this would be a difficult task given the 7 fold increase in payload. The final bridge design did meet the deflection requirement, $1.5 \mathrm{~mm}$ deflection out of the allowable $2.0 \mathrm{~mm}$, and the amount of light obscuration was also deemed acceptable. An interesting figure was presented by the HET design team concerning the original bridge design: the combined mass of the Y Beam (previous nomenclature for the bridge) and carriage (renamed the LHF for the WFU) assemblies accounts for 68 percent of the total tracker's mass ${ }^{5}$. For the WFU, only 52 percent of the total mass consists of the bridge and LHF. While the published stiffness of the HET tracker is higher than the WFU $\left(11.53 \mathrm{~Hz}^{5}\right)$, a 
significantly greater percentage of the tracker's mass was devoted to obtaining this stiffness. Using a similar percentage with the greater WFU payload would result in a heavier tracker requiring substantially higher capacity linear bearings and would have a greater impact to the telescope structure. As it stands, the entire WFU tracker with payload has a mass greater than five times that of the HET tracker.

\section{ACKNOWLEDGMENT}

HETDEX is led by the University of Texas at Austin McDonald Observatory and Department of Astronomy with participation from the Universitäts-Sternwarte of the Ludwig-Maximilians-Universität München, the Max-PlanckInstitut für Extraterrestriche-Physik (MPE), Astrophysikalisches Institut Potsdam (AIP), Texas A\&M University, Pennsylvania State University, and the HET consortium. In addition to Institutional support, HETDEX is funded in part by gifts from Harold C. Simmons, Robert and Annie Graham, The Cynthia and George Mitchell Foundation, Louis and Julia Beecherl, Jim and Charlotte Finley, Bill and Bettye Nowlin, Robert and Fallon Vaughn, Eric Stumberg, and many others, by AFRL under agreement number FA9451-04-2-0355, and by the Texas Norman Hackerman Advanced Research Program under grants 003658-0005-2006 and 003658-0295-2007.

\section{REFERENCES}

[1] Ramsey, L.W., et al., "The early performance and present status of the Hobby-Eberly Telescope,” Proc. SPIE 3352, 34-42 (1998)

[2] Krabbendam, V.L., Sebring, T.A., Ray, F.B. \& Fowler, J.R., "Development and performance of Hobby-Eberly Telescope 11-m segmented mirror,” Proc. SPIE 3352-436 (1998)

[3] Booth, J. A., Ray, F. B., and Porter, D. S., "Development of a star tracker for the Hobby Eberly Telescope," Proc. SPIE 3351-20 (1998)

[4] Hill, G. J., et al., "The Hobby-Eberly Telescope Dark Energy Experiment (HETDEX): Description and Early Pilot Survey Results," ASP Conf. Series, 115-118 (2008)

[5] Savage, R.D., et al., "Current status of the Hobby-Eberly Telescope wide-field upgrade," Proc. SPIE 7733-149 (2010)

[6] Burge, J. H. et al, "Development of a wide-field spherical aberration corrector for the Hobby-Eberly Telescope," Proc. SPIE 7733-51 (2010)

[7] Hill, G. J., MacQueen, P. J., Palunas, P., Shetrone, M. D., Booth, J. A., "Present and Future Instrumentation for the Hobby-Eberly," Proc. SPIE 6269-626907 (2006)

[8] Kelz, A., et al., "Production and performance of replicable integral field units for VIRUS," Proc. SPIE 7735-178 (2010)

[9] Hill, G. J., et al., "VIRUS: a massively replicated 33k fiber integral field spectrograph for the upgraded HobbyEberly Telescope,” Proc. SPIE 7735-21 (2010)

[10] Lee, H., Hill, G. J., Marshall, J. L., DePoy, D. L., Vattiat, B. L., "VIRUS optical tolerance and production,” Proc. SPIE 7735-140 (2010)

[11] Worthington, M. S., Beets, T. A., Good, J. M., Mock, J. R., Murphy, B. T., South, B. J., “Design and development of a high precision, high payload telescope dual drive system,” Proc. SPIE 7733-201 (2010)

[12] Mollison, N. T., et al., "Design and development of a long-travel positioning actuator and tandem constant force actuator safety system for the Hobby-Eberly Telescope wide-field upgrade," Proc. SPIE 7733-150 (2010)

[13] Mock, J. R., Beno, J. H., Zierer, J. J., Rafferty, T. H., Cornell, M. E., "Tracker controls development and control architecture for the Hobby-Eberly Telescope wide field upgrade,” Proc. SPIE 7733-152 (2010)

[14]Zierer, J. Z., Mock, J. R., Beno, J. H., Lazzarini, P. G., Fumi, P., Anaclerio, V., Good, J. M., "The development of high-precision hexapod actuators for the Hobby-Eberly Telescope Wide Field Upgrade," Proc. SPIE 7733-49 (2010)

[15] Kelz, A., et al., "Production and performance of replicable integral field units for VIRUS," Proc. SPIE 7735-178 (2010)

[16] Soukup, I. M., et al., "Design of the fiber optic support system and fiber bundle accelerated life test for VIRUS," Proc. SPIE 7735-180 (2010)

[17]Buckley, D. A. H., Swart, G. P., Meiring, J. G., "Completion and commissioning of the Southern African Large Telescope," Proc. SPIE 6267-62670Z (2006)

[18] Worthington, M. S., "Design and analysis of the Hobby-Eberly Telescope Dark Energy Experiment (HETDEX) bridge," MS thesis, The University of Texas, Austin (2010) 